\title{
Development of Newer Therapies for Non Alcoholic Fatty Acid Liver Disease with Emphasis on Vitamin D and its Receptor and Allyl Isothiocyanate (AITC)
}

\author{
Kulvinder Kochar Kaur ${ }^{1 *}$, Gautam Allahbadia ${ }^{2}$ and Mandeep Singh ${ }^{3}$ \\ ${ }^{1}$ Scientific Director, Dr Kulvinder Kaur Centre for Human Reproduction, Jalandhar, Punjab, India \\ ${ }^{2}$ Scientific Director, Rotunda-A Centre for Human Reproduction, Mumbai, India \\ ${ }^{3}$ Consultant Neurologist, Swami Satyanand Hospital, Jalandhar, Punjab, India \\ *Corresponding Author: Kulvinder Kochar Kaur, Scientific Director, Dr Kulvinder Kaur Centre for Human Reproduction, Jalandhar, \\ Punjab, India.
}

Received: October 14, 2019; Published: November 14, 2019

DOI: $10.31080 /$ ASNH.2019.03.0550

\begin{abstract}
Earlier we have reviewed on lipid metabolism in relation to obesity in the form of role of poly unsaturated fatty acids (PUFA's) and ceramide metabolism in obesity. Further we have been attempting to find ways of tackling nonalcoholic fatty liver disease (NAFLD) which as a result of obesity has attained mammoth proportions. Previously we tried to study roles of leveo carnitine (LC) and nicotiname riboside (NR) having importance in FA $\beta$-oxidation and as a precursor of nicotinamide adenine diamide (NAD+) respectively which acts as a coenzyme in FA beta oxidation to help in reducing the fatty acids from liver. Here we attempted to study further other targets that can be used for treating NAFLD. Utilizing the pubmed search engine we looked for terms like Vitamin D/Vitamin D Receptor (VDR) and allyl isothiocyanate (AITC) in NAFLD, besides other miscellaneous agents like acipimox and curcumin in NAFLD. Of the total 3471 articles we selected a total of 50 articles for this review.
\end{abstract}

Keywords: NAFLD; AITC; Vitamin D/VDR; LC/NR; NAD; Curcumin

\section{Introduction}

All over the world, the prevalence of overweight and obesity in both adults and children has dramatically increased over the last decades and thus become a main health concern. Adiposity along with ectopic fat accumulation are manifestations of obesity which=> comorbidities. Recently non-alcoholic fatty liver disease (NAFLD) has presented to be the most common chronic liver disease that is related to obesity [1,2]. Latest concepts regarding obesity therapy concentrate on lifestyle changes and take into account diet changes advice in combination with exercise. Despite these, obesity rates have not reduced in the last 3 decades. Further recent therapies is insufficient to achieve sustained weight loss for most of the obese patients [3]. Thus need for new strategies which stimulate energy metabolism in other ways is required [4]. Earlier we had reviewed how still we have not achieved any suitable medical therapy for alleviating obesity and comorbidities although thylakoids, GLP1 receptor agonist combinations and some plant products have some promising role and other than Bariatric Surgery we don't have any promising therapy for maintaining weight loss [5-9]. Further we had reviewed regarding role of fat metabolism in effects of obesity and NAFLD development along with role of polyunsaturated fatty acids. Here we further review on how we can attack the lipid metabolism for achieving effective control for obesity and NAFLD [10,11].

\section{Methods}

We used the pubmed search engine for articles regarding NFLD, with special emphasis onMeSH terms like Vit D/Vitamin D receptor and NAFLD, allyl isothiocyanate (AITC) and NAFLD, and other modulators of NAFLD. 


\section{Results and Discussion}

We found a total of 3471 articles of which 90 articles pertaining to Vitamin D/Vitamin D receptor and 60 articles pertaining to AITC and NAFLD, of which we selected 38 articles pertaining to Vitamin D/VDR and14 articles pertaining to AITC and other 8 miscellaneous articles for this review. No meta-analysis was done.

At present nonalcoholic fatty liver disease (NAFLD) is thought to be the most prevalent chronic hepatic disease globally [1]. It affects roughly $1 / 4^{\text {th }}$ of adults globally. From the epidemiological reports it seems that its prevalence is on the rise that say that $25 \%$ right now affect the normal population [2], with a sharp increase in the obese subjects [12], presenting with type2 diabetes mellitus (T2DM) [13], along with the ones with metabolic syndrome (MetS) [14]. Now NAFLD has reached a problem globally, affecting public health [15]. NAFLD basically depicts a broad spectrum of disease stages, varying from simple steasosis to nonalcoholic steatohepatitis (NASH), that has the properties of hepatocellullar damage along with inflammation and ultimately might lead to liver cirrhosis or hepatocellular carcinoma [16]. Moreover NAFLD has a strong tendency to cause T2DM, atherosclerosis, CVS disease along with chronic kidney disease $[17,18]$. With the rapid rise in many countries, it has started becoming an epidemic. Numerous workers have demonstrated a rise in CVS problems, with NAFLD subjects and that NAFLD represents a risk factor that is on its own responsible for >cardiovascular (CVS) mortality [19] (Figure 1).

\section{Role of vitamin D in NAFLD}

Despite the dramatic increase with the clinical implications of this disease what leads to its formation, along with progression is not clear properly and presently no efficacious therapies exist. In the last few yrs some hypothesis given for its aetiopathogenesis have been cited, of which the maximum affected one is the "multiple parallel hits" [20]. As per this hypothesis whatever is influencing accumulation of fat in the liver, inflammation along with fibrosis are influenced by some fine tuning of various causes [20] and related to this vitamin $\mathrm{D}$ and its receptor (VD/VDR) axis is being actively probed. Besides the central part in bone and mineral homeostasis, VD represents a molecule, that has actions on a lot of biological systems, especially the active VD controls the immune system and further affects the insulin sensitivity in different experimental models related to metabolic diseases [21].

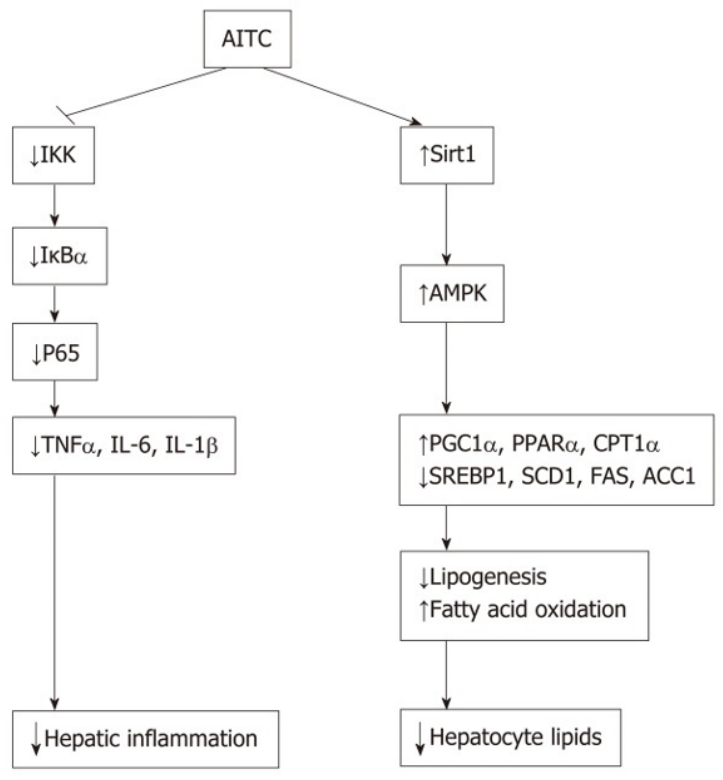

Figure 1: AITC

Courtesy ref no 48 Model of allyl isothiocyanate action. Schematic diagram: allyl isothiocyanate ameliorates hepatic lipid accumulation and hepatic inflammation by activating the Sirt1/AMPK signaling pathway and inhibiting the NF- $\mathrm{\kappa B}$ pathway. AITC: Allyl

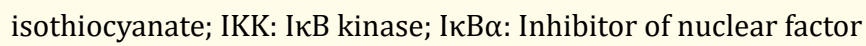
kappa B $\alpha$; TNF $\alpha$ : Tumor necrosis factor $\alpha$; IL-6: Interleukin-6; IL-1 $\beta$ : Interleukin-1 $\beta$; Sirt1: Sirtuin 1; AMPK $\alpha$ : AMP-activated protein kinase $\alpha$; PGC1 $\alpha$ : Proliferator-activated receptor gamma coactivator 1 $\alpha$; PPAR $\alpha$ : Peroxisome proliferator-activated receptor $\alpha$; CPT 1 $\alpha$ : Carnitine palmitoyl transferase $1 \alpha$; SREBP1: Sterol regulatory element binding protein 1; SCD1: Stearoyl coenzyme A desaturase 1; FAS: Fatty acid synthase; ACC1: Acetyl-CoA carboxylase 1.

Lot of researchers have demonstrated that low circulating VD amounts are related to obesity [22], Metabolic Syndrome (MetS) [23], and type 2 diabetes mellitus (T2DM) [15]. Further work done in number of adults showed a good correlation between hypovitaminosis D with the NAFLD diagnosis [25]. Similar results were validated in children, where hypovitaminosis D correlated with histological extreme of NAFLD, that was not dependent on the meta- 
bolic factors [26].

This is further proven by the animal research, that impaired VD balance takes part in the formation of NAFLD. Roth., et al. demonstrated that absence of VD input in obese rats led to the initiation along with progression of NAFLD, that was evaluated via hepatic histology, showing a >NAFLD activity score along with enhancing lobular inflammation [27]. Similarly, in experimental circumstance VD has been demonstrated to possess anti-inflammatory action, along with a marked inhibition of antifibrotic markers expression in the liver, like platelet derived growth factor and transforming growth factor. Further there was decreased production of collagen, $\alpha$-smooth muscle actin along with tissue inhibitorsofmetalloproteinase-1 $\beta$, that represented more anti-inflammatory effects of VD [28]. Another work done on mice having non alcoholic steatohepatitis (NASH), phototherapy decreased inflammation in the hepatocyte along with fibrosis and improved insulin resistance (IR) by enhancing VD.

Based on these proofs given by experiments along with epidemiology, VD has become a centre of interest as a probable treatment for NAFLD. But till now RCTs could not show any improvement with VD addition in decreasing fatty liver amount, or the histological changes showing inflammation and fibrosis, or raised transaminases during the course of either NASH or NAFLD [29].

Hence clinical value of VD in NAFLD remains debatable. Examining the results of randomized controlled trials (RCTs) critically might offer some reasonable basis for carrying out more appropriately designed experiments (like personalized regimens of supplementation related to VD concentrations at baseline and at the point of hepatic damage, >VD supplementation regimens, proportional to VD levels, longer time of supplementation prior to concluding finally with regards to VD use. Still currently one can't see what exact beneficial effects can be got by bringing VD values to

\section{VDR role}

Further part played by VDR has been examined in different metabolic diseases by itself, concentrating mainly on its presence or expression in insulin sensitive tissue including liver. Barchetta., et al. showed for the 1st time in humans the expression of VDR in various liver cell kinds and decreased VDR expression in liver cells of patients having NASH. From that point of time multiple publi- cations revealed that in liver VDR controls microinflammation and fibrosis [30]. Furthermore Arai., et al. [31] showed that in subjects who had biopsy validated NAFLD, polymorphisms of the VDR gene are correlated with the degree of severity of hepatic fibrosis.

Their data revealed that besides VD, secondary hydrophobic bile acids like lithocholic acid, activated VDR in human hepatocytes [32]. In animal studies Bozic., et al. [30], showed that the production of liver steatosis got blunted, once VDR was deleted. When mice were exposed to a HFD, an early induction of liver VDR expression in the presence of fatty liver that was followed by a tendency towards decrease in VDR in the longterm, when> severity of inflammation and fibrosis took place [30]. Same group carried out analysis of expression of genes that were associated with lipid, metabolism in mouse livers, that pointed that lipogenic pathways activation along with inhibition of hepatic oxidation. Furthermore Garcia-Moneizon., et al. [33], showed that liver angiopoietin like protein 8 (ANGPL8) expression is raised on VDR activation. ANGPL8 is recognized as a crucial controller of metabolism of triglycerides and > levels of ANGPL8 is related to the presence of NAFLD [34]. From these results it is indicated that >prominence of VDR is found in liver damage that is in advanced stage, that will point that VDR is induced at an initial stage of the disease and does not need liver injury or fibrosis for having got fully developed.

The total data favours the proposition that, in the period of metabolic diseases, VDR, independent of its own ligand VD might have a key role in helping liver fat accumulation. More studies need to be directed to get full insight in the processes that lead to liver VDR activation and examining the part of a new target for novel strategies in the early tackling of NAFLD.

The pathogenesis of NAFLD is not clear, with no present treatment method or very little options. Beyond life style changes resulting in weight reduction and increased physical activity are the ones that have at present any approval [35]. Thus the requirement of an immediate finding some medical therapies that prove to be efficacious in NAFLD.

\section{Role of allyl isothiocyanate (AITC)}

Increased triglyceride (TG) collecting in the liver hepatocytes is a property of hepatic steasosis, with a marked correlation with chronic hepatic inflammation along with Insulin resistance(IR) 
[36]. Moreover the IkB Kinase (IKK)/nuclear factor kappa B (NFKB) signaling pathways take a key role in the formation of various metabolic disorders which include NAFLD, and particularly hepatic inflammation [37].

Sirtuin 1 (Sirt1) represents a markedly conserved nicotinamide adenine nucleotide-dependent protein deacetylase which controls a broad types of biological actions in mammals which are steasosis along with energy homeostasis [38]. AMP activated protein kinase (AMPK) acts as a switch of energy, regulating various cellular functions like steasosis, via inhibition of lipogenesis in liver and stimulation of fatty acid oxidation [30]. Earlier reports showed that Sirt1 is needed essentially for lipogenesis in liver along with fatty acid oxidation via numerous sensors of nutrients which are sterol regulatory element binding protein1 (SREBP1), peroxisome proliferator activated receptor gamma coactivator $1 \alpha(\mathrm{PGC} 1 \alpha)$ and peroxisome proliferator activated receptor $\alpha$ (PPAR $\alpha)$ [39]. Moreover (Sirt1) controls AMPK activation in NAFLD, causing increased lipolysis and $\beta$-oxidation, and further abrogated hepatic steatosis [41].

Allyl isothiocyanate (AITC) is produced from sinugrin, that is obtained from many cruciferous vegetables, consumes a lot by humans [42]. Myrosinase present within the intestinal organisms, catalyzes the hydrolysis of sinugrin to AITC both in humans along with in animals [42]. Earlier work demonstrated that AITC demonstrated anti- inflammatory in addition to anticancer actions $[43,44]$. AITC was found to be an innovative therapy for DIO along with IR via mitochondrial functioning abnormalities modulation [45]. Further Okulicz M demonstrated that AITC increased both basal along with adrenaline induced lipolysis in adipocytes and augmented the degree of hydrolysis of TG in the blood serum of rats [46]. Further more earlier, Kim., et al. had demonstrated that AITC inhibits adipogenic differentiation of 3T3L1 preadipocytes and repressed gene expression of those that get upregulated at the time of adipogenesis [47]. Still not much answers are there as far as its direct actions on the liver are concerned along with their basic mode of action.

Thus Li., et al. tried to find out the action along with how AITC acted in NAFLD. Thus for developing a mouse along with a cell model of NAFLD, they fed High Fat Diet (HFD) to C57BL/ 6 mice over 8 week period, and treated AML-12 cells with $200 \mu \mathrm{M}$ palmi- tate acid for $12 \mathrm{hrs}$. To receive treatment with AITC, mice were given AITC $(100 \mathrm{mg} / \mathrm{kg} / \mathrm{d})$ via oral route, while AML-12 cells received $20 \mu \mathrm{mol} / \mathrm{L}$ of AITC. With AITC administration HFD induced weight increase was markedly abrogated, along with collection of lipids in the liver and inflammation in vivo. Moreover the transaminases, namely serum alanine amino transferase and aspartate amino transferase levels got significantly decreased in AITC treated mice. As far as mechanism of action is concerned, marked downregulation of SREBP1 along with its lipogenesis genes which it targets, along with upregulaion of proteins participating in FA $\beta$-Oxidation, in addition to upstream mediators like Sirt1 and AMPK $\alpha$ in the livers of HFD mice. Subsequent to AITC administration NFKB signaling pathway got ameliorated. As thought AITC abrogated palmitate acid caused lipid collection and inflammation in AML-12 cells in vitro via the Sirt1/AMPK and NFKB signaling pathways. Interestingly, future work demonstrated that the curative effect of AITC on or lipid collection was ameliorated by siRNA mediated knockdown of either AMPK $\alpha$ or Sirt1 in AML-12 cells. Thus concluding that marked abrogation of hepatic steatosis and inflammation was caused by AITC via activation of Sirt1/AMPK and NFKB pathway.

\section{Role of acipimox}

IR is related to enhanced lipolysis and increased concentration of FFA $s$ that ultimately result in compromised vascular function. Aday., et al. proposed that decreasing FFA with acipimox, that is a derivative of nicotinic acid, which compromises FFA efflux, might help in increasing endothelial function as observed by flow-mediated dilation, in subjects with MetS. They recruited 18 patients having MetS along with 17 healthy controls and gave them $250 \mathrm{mg}$ acipimox orally $6 \mathrm{hrly} /$ placebo for 7 days utilizing a randomized double blind, crossover trial. They observed that acipimox decreased amounts of Free Fatty Acid (FFA) in cases of MetS to approximately normal concentrations $(\mathrm{p}=0.01)$, without any changes found in healthy controls ( $\mathrm{p}=0.17)$. No improvement of endothelial - based FMD was found in any group (MetS; $\mathrm{P}=0.42$; Healthy Controls: $\mathrm{p}=0.16$ ) by acipimox, though endothelial nondependent nitroglycerin -mediated dilation in cases presenting with MetS had a tendency of rising $(20.3 \%, p=0.06)$. No differences in lipids or inflammation markers were seen after therapy. Very little alterations in FMD along with baseline BMI measures $(p=-0.09)$ or waist circumference $(\mathrm{p}=-0.15)$. Thus concluding that in those having normal or increased baseline FFA, short time decreases don't enhance endothelial function as studied using FMD [49].

Citation: Kulvinder Kochar Kaur., et al. "Development of Newer Therapies for Non Alcoholic Fatty Acid Liver Disease with Emphasis on Vitamin D and its Receptor and Allyl Isothiocyanate (AITC)". Acta Scientific Nutritional Health 3.12 (2019): 154-160. 


\section{Role of curcumin}

The main risk factor for MetS development is obesity, that refers to the pathological hyperplasia or/and hypertrophy of AT, How many mature adipocytes will be present is on the bases of differentiation of adipocytes from preadipocytes. Wu., et al. tried to examine the actions of curcumin on adipogenesis and its mechanism. For testing cell toxicity caused by curcumin, 3T3L1 preadipocytes got $0-50 \mu \mathrm{M}$ curcumin for a period of $24 \mathrm{~h}, 48 \mathrm{~h}$ or $72 \mathrm{~h}$, and then they measured cell viability with the help of MTT (3- (4, 5-dimethyl thiazol-2-yl) 2, 5-diphenyl tetrazolium bromide) assay. Curcumin's action on cell cycle was measured by flow cytometry. Cell apoptosis stimulated by curcumin was checked by the TUNEL Assay and caspase activation was checked by immunoblotting. Effect of curcumin on the differentiation of adipocytes was checked using mitotic clonal expansion (MCE), expression of adipogenic transcription factors, along with lipid collection. Marked reduction of viability of preadipocytes was observed following therapy with $30 \mu \mathrm{M}$ curcumin, that is an amount that results in apoptosis in preadipocytes, as checked, using TUNEL assay, and lead to activation of caspases 8,9 along with 3 . Non cytotoxic dose of curcumin $(15 \mu \mathrm{M})$ inhibited MCE, along with the downregulation of the expressionof PPAR $\gamma$ and $\mathrm{C} / \mathrm{EBP} \alpha$, disallowed differentiation -medium stimulated $\beta$-catenin downregulation, and reduced lipid collection in 3T3L1 adipocytes. Thus concluding that curcumin can stimulate preadipocytes apoptosis and prevent adipocytes differentiation $=>$ reduction of adipogenesis [50].

\section{Conclusion}

Earlier we had reviewed regarding importance of polyunsaturated fatty acids and role of ceramide metabolism in obesity and utilization of Levo Carnitine and Nicotnamide Riboside combination in treating NAFLD. Here we have further emphasized on role of Vitamin D although adding vitamin D has not clearly yielded results of resolution of NAFLD, needing further studies, and role of Vitamin D receptor irrespective of its ligand Vitamin D in liver which needs to be further investigated for its role in NAFLD, roles of acipimox and curcumin are further explored.

\section{Bibliography}

1. Younossi ZM., et al. "Global burden of NAFLD and NASH: Trends, predictions, risk factors and prevention". Nature Reviews Gastroenterology and Hepatology 15 (2018): 11-20.

2. Younossi ZM., et al. "The economic and clinical burden of nonalcoholic fatty liver disease in the United States and Europe". Hepatology 64 (2016): 1577-1586.
3. Fildes A., et al. "Probability of an obese person attaining normal weight: Cohort using study using electronic health records". American Journal of Public Health 105.9 (2015): 54-59.

4. Lee Y., et al. "Dietary isoliquiritigenin at a low dose ameliorates insulin resistance and NAFLD in diet induced obesity in C57BL/6J mice". International Journal of Molecular Sciences 19 (2018): 3281.

5. Kulvinder Kochar Kaur., et al. "Existing and prospective pathways for intervention in treatment of obesity in a novel way-a review". MOJ Drug Design Development and Therapy 2 (2018): 95-105.

6. Kulvinder Kochar Kaur., et al. "Can Thylakoids Replace Bariatric Surgery for Long Term Maintenance of Weight Loss in Obesity Giving A More Physiological Approach". Obesity Control Therapy 5 (2018): 1-10.

7. Kulvinder Kochar Kaur., et al. "Are we at the verge of finding a new efficacious pharmacotherapy for obesity in the form of agonism at triple drug receptors: glucagon, Glucagon like peptide1 (GLP1), glucose dependent insulin tropic peptide (GIP)". International Physical Medicine and Rehabilitation Journal 3 (2019): 22-27.

8. Kulvinder Kochar Kaur., et al. "An Update on Bariatric Surgery with Long Term Efficacy and Its Utilization for Medical Therapy Development from the Different Mechanism of Action and Other Short Comes to Be Outcome". BAOJ Surgery 4 (2018).

9. Kulvinder Kochar Kaur., et al. "Role of Natural Products in the Treatment of Diabesity with Mechanism of Action-A Small Communication". Acta Scientific Nutritional Health 3.7 (2019): 140-142.

10. Kulvinder Kochar Kaur., et al. "Review of Nutrient Metabolism in Obesity with Special Emphasis on Fatty Acid Metabolism". BAOJ Food Science and Technology 1 (2017).

11. Kulvinder Kochar Kaur., et al. "Synthesis and functional significance of Poly Unsaturated fatty acids (PUFA's) in body". Acta Scientific Nutritional Health 2 (2018): 43-50.

12. Leonardo A., et al. "Epidemiological modifiers of nonalcoholic fatty liver disease. Focus on high risk groups". Digestive and Liver Disease 47 (2015): 997-1006.

13. Targher G., et al. "Prevalence of nonalcoholic fatty liver disease and its association with cardiovascular disease among type 2 diabetics". Diabetes Care 30 (2007): 1212-1218. 
14. Yki - Jarvinen H. "Nonalcoholic fatty liver disease as a cause and consequence of metabolic syndrome". Lancet Diabetology and Endocrinology 2 (2014): 901-910.

15. Estes C., et al. "Modeling the epidemic of nonalcoholic fatty liver diseas demonstrates an exponential increase in burden of disease". Hepatology 67 (2018): 123-133.

16. Cohen JC., et al. "Human fatty liver disease: old questions and new insights". Science 332 (2011): 1519-1523.

17. Chalasani N., et al. "The diadnosis and management of nonalcoholic fatty liver disease:practice Guidelines by the American Association of the Study of Liver Diseases, American College of Gastroenterological Association". Hepatology 55 (2012): 2005-2023.

18. Sinn DH., et al. "Persistent nonalcoholic fatty liver disease increases risk for Carotid Atherosclerosis". Gastroenterology 151 (2016): 481-488.

19. Mahfoud-Haddad T., et al. "Nonalcoholic fatty liver disease and the risk of clinical cardiovascular events: A Systematic review and Meta-analysis". Diabetes Metab Syndr 11 (2017): S209-S216.

20. Tilg H and Moschen AR. "Evolution of inflammation and nonalcoholic fatty liver disease: The multiple parallel hits hypothesis". Hepatology 52 (2010): 1836-1846.

21. Elseweidy MM., et al. "Nonalcoholic fatty liver disease intake as regulator of insulin degradating enzymes and insulin receptor phosphorylation in diabetic rats". Biomed Pharmacotherapy 85 (2017): 185-89.

22. Bell NH., et al. "Evidence for alteration of the Vitamin D endocrine system in obese subjects". Journal of Clinical Investigation 76 (1985): 370-375.

23. Barchetta I., et al. "Hypovitaminosis D is independently associated with metabolic syndrome in obese patients". PLoS One 8 (2013): e68689.

24. Boucher BJ. "Vitamin D insufficiency and diabetic rats". Current Drugs Targets 12 (2011): 61-87.

25. Wang D., et al. "Vitamin D levels are inversely inversely associated with liver fat content and risk of nonalcoholic fatty liver disease in Chinese middle aged elderly population: The Shanghai Changing Study". PLoS One 11 (2016): e0157515.
26. Manco C., et al. "Low levels of 25-hydroxy vitamin D (3) in children with biopsy proven nonalcoholic fatty liver disease". Hepatology 51 (2010): 2229.

27. Ruth CL., et al. "Vitamin D deficiency in obeserats exacerbates nonalcoholic fatty liver disease and increases hepatic resistin and Toll like receptor activation". Hepatology 55 (2012): 11031111.

28. Beilfuss A., et al. "Vitamin D counteracts fibrogenic TGF $\beta$ signaling in human hepatic stellate cells both receptor -dependently and independently". Gut 64 (2015): 791-799.

29. Lorvand Amiri H., et al. "Effect of daily calcitrio supplementation with and without calcium on disease regression in nonalcoholic fatty liver patients following an energy restricted diet:Randomized, controlled, double blind trial". Clinical Nutrition 36 (2017): 1490-1497.

30. Bozic M., et al. "Hepatocyte Vitamin D receptor regulates lipid metabolism and mediates experimental diet induced steatosis". Journal of Hepatology 65 (2016): 748-757.

31. Arai T., et al. "Association of Vitamin D levels and Vitamin D-related gene polymorphisms with liver fibrosis in patients with biopsy proven nonalcoholic fatty liver disease". Digestive Liver Disease 51 (2019): 1036-1042.

32. Han S., et al. "Anovel bile acid activated vitamin D receptor signaling in human hepatocytes". Molecular Endocrinology 24 (2010): 1151-1164.

33. Garcia -Monzon., et al. "Angiopoietin -Like Protein 8 is a novel vitamin D receptor Target gene involved in nonalcoholic fatty liver pathogenesis". American Journal of Pathology 188 (2018): 2800-2810.

34. Lee YH., et al. "Association between betalipotrophin/ANGPTL8 and nonalcoholic fatty liver disese: Animal and human studies". Science Report 6 (2016): 24013.

35. Younossi ZM., et al. "Current and future therapeutic regimens for nonalcoholic fatty liver disease and nonalcoholic steatohepatitis". Hepatology 68 (2018): 361-371.

36. Samuel VT and Shulman GI. "Mechanisms for insulin resistance: common threads and missing link". Cell 148 (2012): 852-871. 
37. Wang PX., et al. "Interferon regulatory factor 3 constrains IKK $\beta / \mathrm{NF \kappa B}$ signaling to alleviate hepatic steatosis and insulin resistance". Hepatology 59 (2014): 870-885.

38. Picard F., et al. "Sirt1 promotes fat mobilization in white adipocytes by expressing PPAR-gamma". Nature 429 (2004): 771-776.

39. Herzig S and Shaw RJ. "AMPK: guardian of metabolism and mitochondrial homeostasis". Nature Reviews Molecular Cell Biology 19 (2018): 121-135.

40. Li Y., et al. "Hepatic SIRT1 attenuates hepatic steatosis and controls energy balance in mice by inducing fibroblast growth factor 21". Gastroenterology 146 (2014): 539-49e7.

41. Dogra S., et al. "Zinc oxide nanoparticles attenuates hepatic steatosis development in high fat diet mice through activated AMPK signaling axis". Neuroendocrine 17 (2019): 210-222.

42. Zhang Y. "Allyl isothiocyanate as a cancer chemopreventive phytochemical". Molecular Nutrition and Food Research 54 (2010): 127-135.

43. Xiao D., et al. "Allyl isothiocyanate, a constituent of cruciferous vegetables inhibits proliferation of human prostate cancer cells by causing G2/M arrest and inducing apoptosis". Carcinogenesis 24 (2003): 891-897.

44. Wagner AE., et al. "Antiinflammatory potential of Allyl isothiocyanate -role of Nrf2, NFKB and microRNA-155". Journal of Cellular and Molecular Medicine 16 (2015): 836-845.

45. Ahn J., et al. "Allyl isothiocyanate ameliorates insulin resistance through the regulation of mitochondrial function". Journal of Nutritional Biochemistry 25 (2014): 1026-1034.

46. Okulicz M. "Multidirectional time-dependent effect of sinigrin and Allyl isothiocyanate on metabolic parameters in rats". Plant Foods for Human Nutrition 25 (2014): 1026-1034.

47. Kim YJ., et al. "Pharmacokinetics, Tissue Distribution, and Anti -Lipogenic effects of Allyl isothiocyanate matabolites". PLoS One 10 (2015): e0132151.
48. Li CX., et al. "Allyl isothiocyanate ameliorates lipid accumulation and inflammation in nonalcoholic fatty liver disease via the Sirt1/AMPK and NFKB signaling pathways". World Journal of Gastroenterology 25 (2019): 5120-5133.

49. Aday AW., et al. "Impact of Acipimox on Free Farry Acid Efflux and Endothelial Function in the Metabolic Syndrome: A Randomized Trial". Obesity (Silver Springs) (2019).

50. Wu LY., et al. "Curcumin Attenuates Adipogenesis by Inducing Preadipocyte Apoptosis and Inhibiting Adipocyte Differentiation". Nutrients 11 (2019): pii:E2307.

\section{Volume 3 Issue 12 December 2019}

(C) All rights are reserved by Kulvinder Kochar Kaur., et al. 